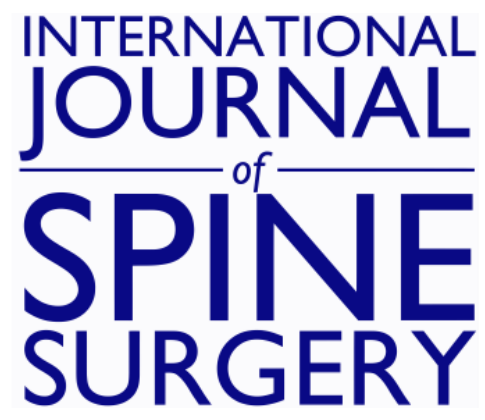

\title{
Range of Motion of the Intact Lumbar Segment: A Multivariate Study of 42 Lumbar Spines
}

Daniel J. Cook, Matthew S. Yeager and Boyle C. Cheng

Int J Spine Surg 2015, 9 ()

doi: https://doi.org/10.14444/2005

http://ijssurgery.com/content/9/5

This information is current as of April 26, 2023.

Email Alerts Receive free email-alerts when new articles cite this article. Sign up at:

http://ijssurgery.com/alerts

The International Journal of Spine Surgery

2397 Waterbury Circle, Suite 1,

Aurora, IL 60504, Phone: +1-630-375-1432

(C) 2015 ISASS. All Rights,Refserded 


\section{Range of Motion of the Intact Lumbar Segment: A Multivariate Study of 42 Lumbar Spines}

Daniel J. Cook, MS, Matthew S. Yeager, BS, Boyle C. Cheng, PhD

Department of Neurosurgery, Allegheny Health Network, Pittsburgh, PA

\section{Abstract}

\section{Background}

A thorough understanding of the biomechanical characteristics of the healthy human spine is critical in furthering the treatment of spinal pathology. The goal of this study was to investigate the motion of the intact lumbar spine segment as measured by range of motion (ROM), and to investigate the dependencies thereof on gender and intervertebral level.

\section{Materials and Methods}

Kinematic data was obtained for 42 human lumbar segments (L1-S1) in response to a pure-moment loading protocol in flexion extension (FE), lateral bending (LB) and axial torsion (AT). Data was obtained for 204 individual functional spinal units (91 female, 113 male). Multivariate analysis of variance was conducted to detect differences between genders and intervertebral levels in each mode of loading. Correlations between ROM and donor demographics, including height, weight, and age, were conducted.

\section{Results}

ROM was significantly greater for females than for males in FE, LB and AT ( $p<0.001)$. ROM tended to increase down the vertebral column in FE. L3-4 FE ROM was significantly greater than L1-2 ( $\mathrm{p}=0.024)$, and L4-5 and L5-S1 FE ROM were significantly greater than for every other level $(\mathrm{p}<0.003)$. LB ROM tended to be greater toward the center of the segment with L2-3, L3-4 and L4-5 ROM being significantly greater than both L1-2 $(\mathrm{p}<0.001)$ and L5-S1 ( $\mathrm{p}=0.006, \mathrm{p}<0.001, \mathrm{p}=0.043$, respectively). A similar trend was found for AT, however only L1-2 was significantly less than all other levels $(\mathrm{p}=0.042, \mathrm{p}<0.001, \mathrm{p}<0.001$, and $\mathrm{p}=0.034$ for L2-3, L3-4, L4-5, and L5-S1 respectively).

\section{Conclusion}

The significant differences in lumbar ROM between male and female spine segments and between the intervertebral levels must be taken into account in study design in order to prevent biases in outcomes. The significant differences in ROM between levels may also have critical implications in the design of spinal implants, particularly those designed to maintain or restore healthy motion.

KEYWORDS: SPINE BIOMECHANICS, INTACT SPINE, RANGE OF MOTION, LUMBAR SPINE

VOLUME 9 ARTICLE 5 DOI: 10.14444/2005

\section{Introduction}

A thorough understanding of the biomechanical characteristics of the healthy human spine is critical in furthering the treatment of spinal pathology. The rotational range of motion (ROM) in each of the primary modes of loading, flexion extension (FE), lateral bending (LB), and axial torsion (AT), is the most commonly used and most widely understood metric describing the biomechanical behavior of the spine.
Descriptive statistics of healthy spine ROM and its relationship to demographic variables, such as gender, age, weight, and height, as well as morphometric variables, such as intervertebral level, may provide critical input to implant design, biomechanical study design and interpretation, development of diagnostic criteria, and the design of biomechanical models of the spine. 
Most in vitro biomechanical studies of the spine include the unaltered osteoligamentous structures, commonly referred to as the "Intact" condition, as the primary treatment group for comparison. This is a convenient way to control for variability between specimens and to provide a meaningful interpretation of study results at the same time. Given the difficulty in defining "healthy" in any objective sense, the Intact spine segment, derived from donors without a noted history of treatment for spinal disorders, is a reasonable proxy for the behavior of the healthy spine.

Several attempts have been made to characterize spine segment motion in living subjects as well as in cadaveric models. In 1978, White and Panjabi provided a comprehensive summary of the available literature in an attempt to provide an estimate of typical motion at each intervertebral level. ${ }^{1}$ Citing differences in experimental techniques and the broad range of biologic variability, the authors provided boundaries for ROM at each level along with a representative angle, which constituted their best opinion based on the review of the literature and their own analysis. Many cadaveric studies have been published which include the ROM of the Intact segment, usually in comparison to one or more surgical treatments on relatively few specimens (6-10). On the other hand, several notable studies have been conducted on the Intact lumbar segment and the relationship between ROM and various contributing factors, such as age, gender, intervertebral level, and degenerative condition, with sample sizes ranging from 42 to 110 individual motion segments. ${ }^{2-5}$

The goal of this study was to investigate the motion of the intact lumbar spine segment as measured by ROM, and to investigate the dependencies thereof on gender and intervertebral level. The data collected from the Intact treatment condition across six biomechanical studies, conducted in the same laboratory under the same conditions, were combined and analyzed.

\section{Materials and Methods}

Data from the Intact treatment of six separate lumbar biomechanical studies, conducted in a single labora- tory under the same specimen preparation and testing protocol and by the same staff, were compiled for this study. The full data sets from some of these studies have been previously published, while the remaining are not yet published. ${ }^{6-8}$ Kinematic data was obtained for 42 human cadaveric lumbar segments (L1-S1) in response to a pure-moment loading protocol in flexion extension (FE), lateral bending (LB) and axial torsion (AT). A six-degree-of-freedom spine tester (Bose, Smart Test Series, Eden Prairie, $\mathrm{MN}$ ) was utilized for all testing. The spine tester is shown in Figure 1 and consists of counteracting FE and LB gimbals mounted within a pneumatically controlled biaxial test frame. All modes of loading consisted of three cycles of sinusoidal loading to limits of $\pm 7.5 \mathrm{~N} \mathrm{~m}$ at a frequency of $0.005 \mathrm{~Hz}$. The third cycle of each testing mode was used for analysis, with the first two intended for preconditioning of the segment. Four infrared light-emitting diodes (LED), inserted within precision-machined aluminum tracking bodies, were affixed to the anterior surface of each vertebral body as shown in Figure 2. The location and orientation of each vertebra was monitored, using the attached LED, at $10 \mathrm{~Hz}$ throughout testing using an Optotrak Certus motion capture system (Optotrak, Northern Digital Instruments, Waterloo, ON, Canada) with a manufacturer stated accuracy of $0.1 \mathrm{~mm}$.

Complete data was obtained for 204 individual functional spinal units (FSU) of which 91 were from female donors and 113 were from males. Table 1 includes the descriptive statistics for age, height and weight of the donors used in this study. Though all segments were fixed to the loading apparatus in the same fashion, kinematic data for $\mathrm{S} 1$ was not tracked during one of the studies, so L5-S1 data was only collected on 36 of the lumbar segments.

Local coordinate systems were defined for each vertebral tracking body based on the location of the LED contained within it. The $\mathrm{x}$-axis was defined as oriented to the right side of the specimen when viewed from the posterior. The y-axis was defined as normal to the plane containing the four LED, facing anteriorly. The z-axis was defined as mutually orthogonal to the $\mathrm{x}$ - and $\mathrm{y}$-axes, directed superiorly. Euler angles were calculated from the transformation matrices de- 
scribing the relative position and orientation of each vertebral tracking body to its inferior neighbor in the sequence Xy'z". FE ROM was defined as the range of the first Euler angle ( $\alpha$, corresponding to the $\mathrm{X}$ axis) during the third cycle of FE loading. LB ROM was defined as the range of the second Euler angle ( $\beta$, corresponding to the $y^{\prime}$ axis) during the third cycle of LB loading. AT ROM was defined as the range of the third Euler angle ( $\gamma$, corresponding to the $z$ " axis) during the third cycle of AT loading.

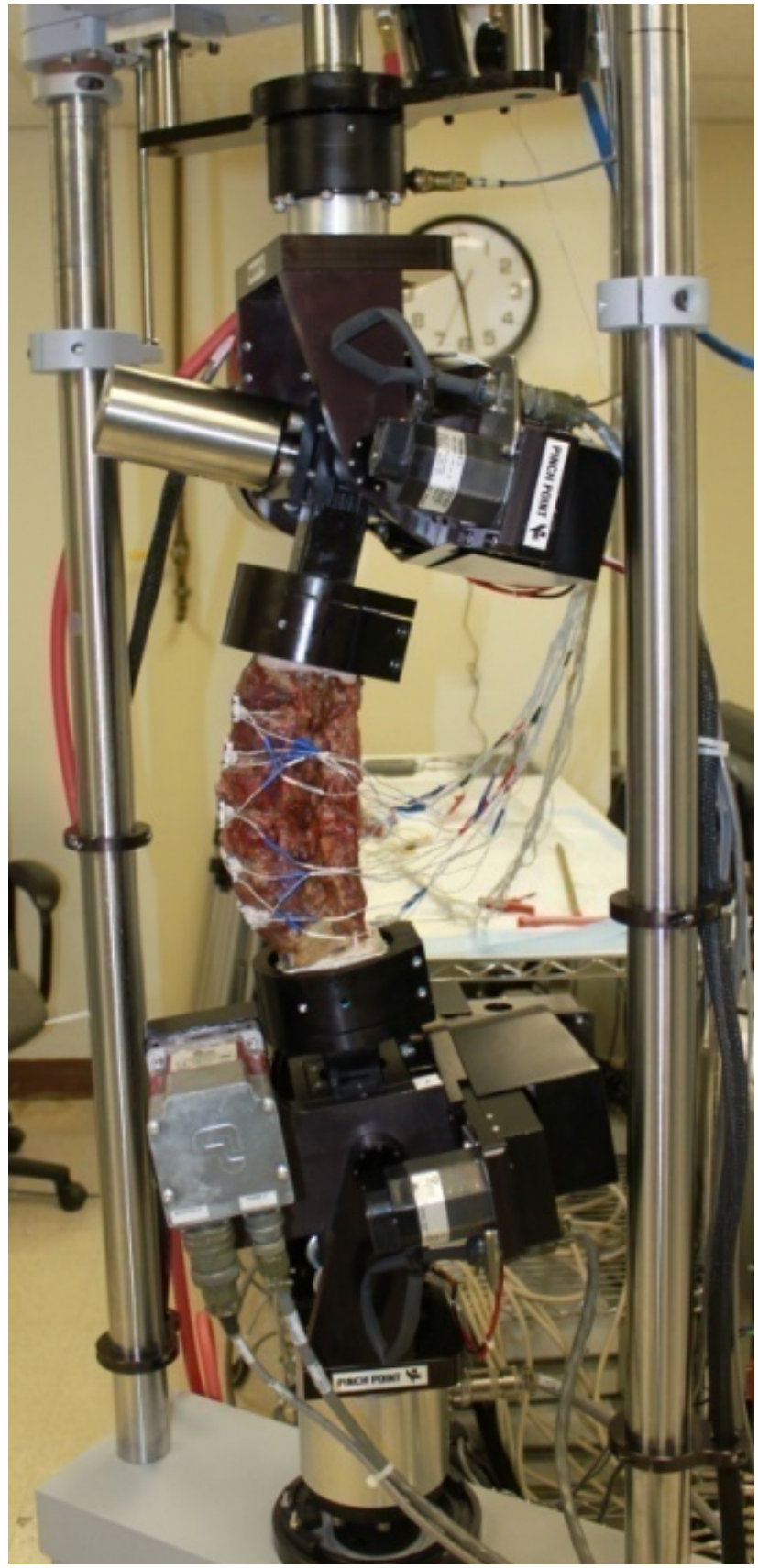

Fig. 1. Spine Testing Apparatus with Lumbar Segment. T12 and Sacrum are set within potting resin and fixed to the testing apparatus.

\section{Statistical Methods}

Multivariate analysis of variance with donor age, height, and weight as covariates, and with Bonferroni post-hoc comparison, was conducted to detect differences between genders and intervertebral levels in each mode of loading. Correlations between ROM in each mode and donor demographics, including height, weight and age were also conducted. All statistical analysis was conducted in SPSS (Version 20, IBM, Armonk, New York). All presented p-values have had the appropriate Bonferroni correction factor applied so that proper interpretation of significance still relies on the acceptance criterion of $\mathrm{p}<0.05$.

\section{Results}

ROM was significantly greater for females than for males in FE, LB and AT ( $\mathrm{p}<0.001)$. When comparing between males and females within intervertebral levels, females exhibited significantly greater ROM at every level in FE $(\mathrm{p}<0.005), \mathrm{LB}(\mathrm{p}<0.003)$ and in AT $(\mathrm{p}=0.004, \mathrm{p}<0.001, \mathrm{p}<0.001, \mathrm{p}<0.001, \mathrm{p}=0.011$ for L1-2, L2-3, L3-4, L4-5 and L5-S1 respectively). These comparisons are shown in Figure 3, Figure 4 and Figure 5. In FE, ROM of Female specimens

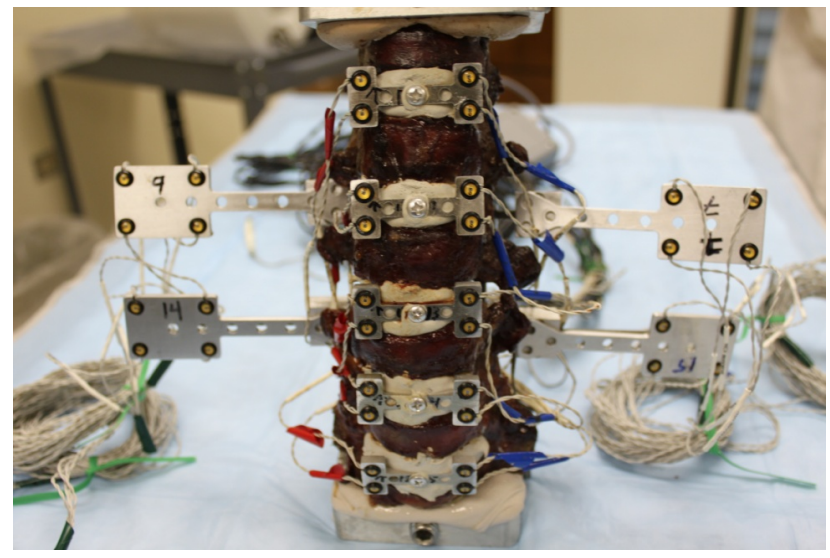

Fig. 2. Lumbar Segment with LED Tracking Bodies Attached to Anterior Surface. Local coordinate axes were defined for each vertebra with $x, y$ and $\mathrm{z}$ axes oriented in the general directions depicted to the right.

Table 1. Donor Descriptive Statistics
\begin{tabular}{|l|r|r|r|r|}
\hline & Minimum & Maximum & Mean & Std. Deviation \\
\hline Age (years) & 24 & 74 & 57.52 & 9.29 \\
\hline Height (in) & 59 & 75 & 66.75 & 4.11 \\
\hline Weight (lbs) & 90 & 400 & 215.32 & 79.65 \\
\hline
\end{tabular}


ranged from $23 \%$ to $34 \%$ greater mean ROM than Male specimens at L3-4 and L1-2, respectively. In LB, ROM of Female specimens ranged from $20 \%$ to 53\% greater at L3-4 and L4-5, respectively. The largest comparative differences were seen in AT, in which Female segments exhibited greater mean ROM ranging from $41 \%$ greater at L5-S1 to $96 \%$ greater at L4-5.

ROM tended to increase down the vertebral column in FE. L3-4 FE ROM was significantly greater than L1-2 ( $p=0.024)$, and L4-5 and L5-S1 FE ROM were

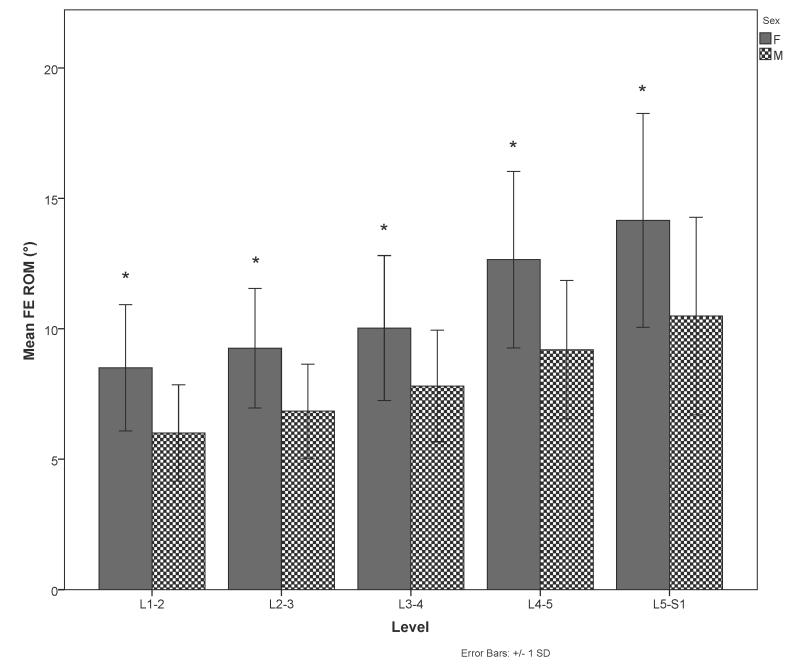

Fig. 3. FE ROM Gender Comparisons within Intervertebral Level (* Indicates significant difference between males and females at the indicated intervertebral level)

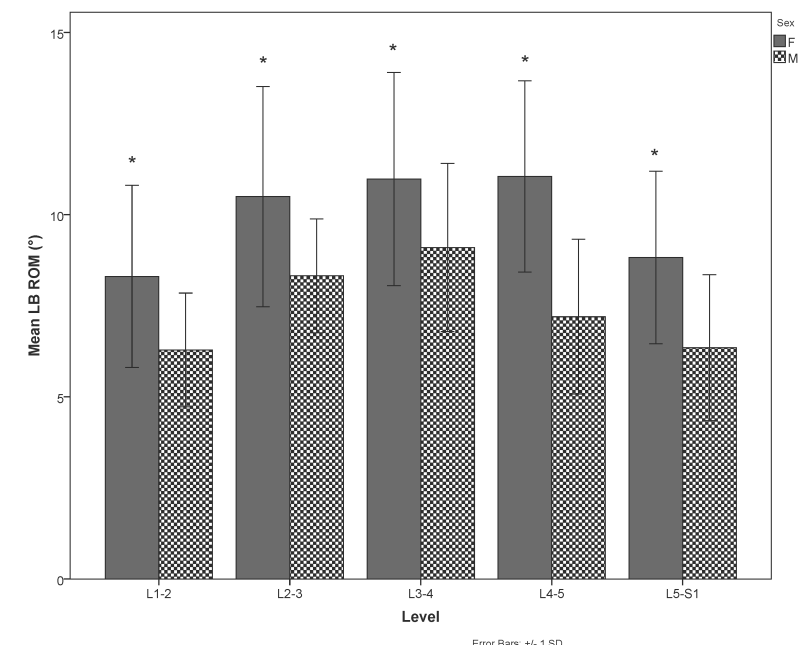

Fig. 4. LB ROM Gender Comparisons within Intervertebral Level (* Indicates significant difference between males and females at the indicated intervertebral level) significantly greater than for every other level $(\mathrm{p}<0.003)$. LB ROM tended to be greater toward the center of the segment with L2-3, L3-4 and L4-5 ROM being significantly greater than both L1-2 $(\mathrm{p}<=0.001)$ and L5-S1 ( $\mathrm{p}=0.006, \mathrm{p}<0.001, \mathrm{p}=0.043$, respectively). A similar trend was found for AT, however only L1-2 was significantly less than all other levels $(\mathrm{p}=0.042, \mathrm{p}<0.001, \mathrm{p}<0.001$, and $\mathrm{p}=0.034$ for L2-3, L3-4, L4-5, and L5-S1 respectively).

A significant negative correlation was found between FE ROM and height $(r=-0.223, p=0.001)$ as well as between FE ROM and weight $(r=-0.382, p<0.001)$. A significant negative correlation was also found between LB ROM and height $(r=-0.222, p=0.001)$ and between LB ROM and weight $(r=-0.430, p<0.001)$. A significant positive correlation was found between AT ROM and age $(\mathrm{r}=0.273, \mathrm{p}<0.001)$. A significant negative correlation was found between AT ROM and weight $(\mathrm{r}=-0.280, \mathrm{p}<0.001)$.

\section{Discussion}

While there have been many studies published which include the ROM of the Intact spine, they are usually designed with the intent of evaluating one or more fixation or motion preservation implants in relation to the Intact or a Destabilized condition. ${ }^{9}$ Thus, the sample size of any single study is usually limited to 6-10 spines, which precludes well-powered comparisons between demographic variables or between in-

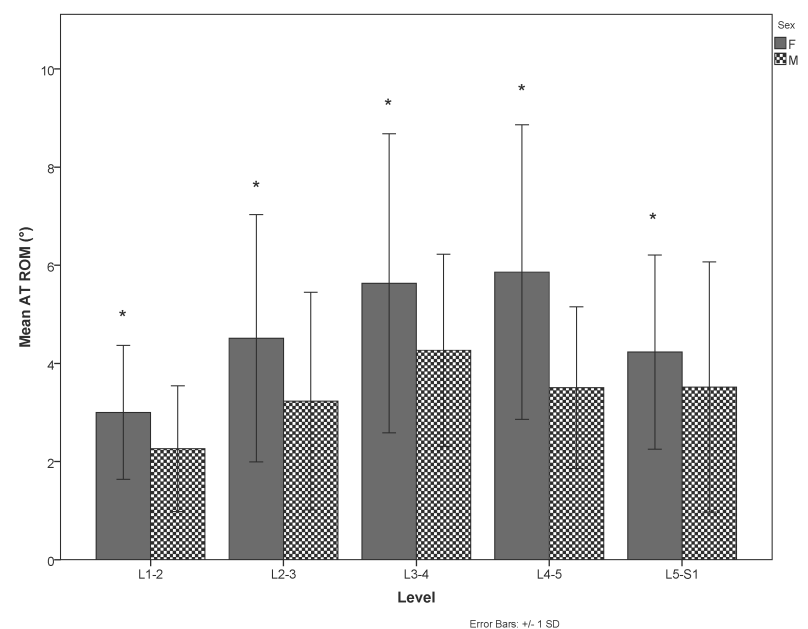

Fig. 5. AT ROM Gender Comparisons within Intervertebral Level (* Indicates significant difference between males and females at the indicated intervertebral level) 
tervertebral levels. ${ }^{10,11}$ Furthermore, different intervertebral levels, e.g. L1-S1, L3-S1, may be included in a given study depending on the objectives of the study, the availability of tissue and the equipment of the testing facility. While pure-moment loading should ameliorate dependency of in-plane loads on segment length compared to other methods of loading, the length of the tested segments may influence the degree of lordosis exhibited and thus the coupling behavior between LB and AT. ${ }^{12}$ Therefore, comparisons between studies must take this factor into account. In addition, the testing equipment used varies considerably between laboratories, ${ }^{13}$ with a great deal of the published data derived from systems relying on manually operated weight and pulley systems,${ }^{2,14,15}$ some relying on automated pulley-cable systems, ${ }^{4,5,16}$ and others utilizing electromechanical or servo-hydraulic systems. ${ }^{13}$ Because of differences in study design and laboratory testing techniques, compilation of Intact data sets across the published literature would be a difficult task, potentially fraught with error. In the current study, the authors have had the ability to combine the Intact data derived from several different studies under precisely the same testing conditions, leading to the largest known data set of its kind. The inter-level and inter-gender differences found in the current study seem to support much of the previous work done on the Intact spine.

Nachemson et al. evaluated 42 cadaveric functional spinal units (FSU) with respect to age, sex, intervertebral level and degenerative condition. ${ }^{2}$ The group found no influence of age or intervertebral level on ROM but did note that female segments appeared to be more flexible than males, as was found in the current study. No statistical comparisons between genders or degenerative classes were provided however, and the group noted that individual differences between motion segments overshadowed differences between classes. Mimura et al. studied 47 lumbar FSU, derived from 12 male specimens, and reported statistically significant decreasing LB ROM and increasing AT ROM with degeneration. ${ }^{4}$ The authors also found significant increases in joint laxity associated with degeneration, but unfortunately no intergender comparisons could be made within the allmale cohort. Yamamoto et al. conducted a study of 10 lumbar spine segments (L1-Sacrum), finding in- creasing FE ROM at lower levels (L4-5 and L5-S1) and maximum LB ROM at L2-3. These results appear to support the greater FE ROM found at the lower lumbar levels in the current study. However, no statistical comparisons were made between intervertebral levels, and no comparisons were made between genders. Fujiwara et al. conducted a study on 110 FSU from 44 specimens to determine the effects of disc degeneration and facet osteoarthritis on lumbar spine flexibility. Further supporting the results of the current study, the group reported significantly greater ROM for female segments compared to males. The authors also noted differing patterns of influence of disc and facet degeneration on ROM between males and females.

Several in vivo studies have been conducted to investigate the ROM of the symptomatic and asymptomatic human lumbar spine. ${ }^{17-22}$ Such studies typically involve standing or lying lateral and/or anterior-posterior radiographs in order to assess $\mathrm{FE}$ and/or LB ROM. The reported mean values from several in vivo studies are provided in Figure 6 and Figure 7 along with the mean values reported by Yamamoto et al. and the mean values found in the current study. Considerable variability exists between the values reported for each study. Several factors may contribute to this variability including differing study populations and study procedures as well as uncertainty associated with radiographic measurements themselves. A summary of the study population for each of the referenced articles is shown in Table 2. In spite of the considerable variability in reported FE ROM, both from in vivo and in vitro studies, the data appear to support the finding of increased FE ROM down the lumbar vertebral column as shown in Figure 6. Further, the data presented in the current study appears to be comparable to the lower end of the range of data presented in the literature. Unfortunately, most studies of the in vivo spine did not include measurement of LB ROM. Figure 7 includes summary LB statistics reported by Dvorak, Tanz, and Yamamoto in comparison to the results from the current study. Considerable disagreement is demonstrated between studies, particularly at L5-S1, precluding conclusive corroboration of the current study with reported literature with respect to LB ROM. Several factors may contribute to the differences seen 
between LB ROM in in vivo and in vitro studies. Two-dimensional radiographic imaging of coronal plane motion is particularly subject to uncertainty given the lordotic curvature of the lumbar spine, and given the coupled, out-of-plane motion that occurs during lateral bending. These challenges are not present in in vitro studies relying on three-dimensional motion capture. Also, differences may result from incongruence between pure-moment loading conditions applied in the laboratory and the physiologic loads present in test subjects.

Several significant correlations were found between intervertebral ROM and donor demographic information, including height, weight and age. However, despite the presence of statistically significant correlations, linear regression between ROM and these variables yields very low predictive value $\left(\mathrm{R}^{2}=0.049-0.185\right)$. Despite the inclusion of age, height and weight as covariates in the analysis, significant differences between male and female segments

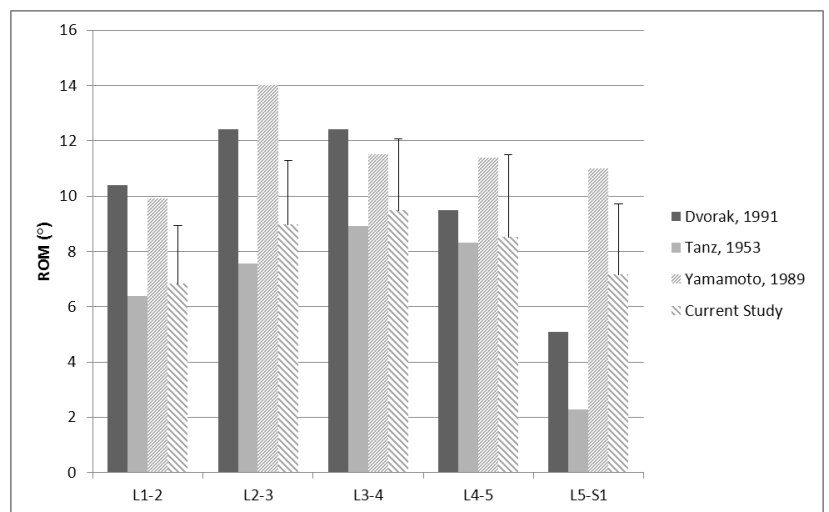

Fig. 6. FE ROM Literature Values Compared to Current Study. Striped bars signify in vitro studies, while shaded ones represent in vivo. Error bars represent standard deviation.

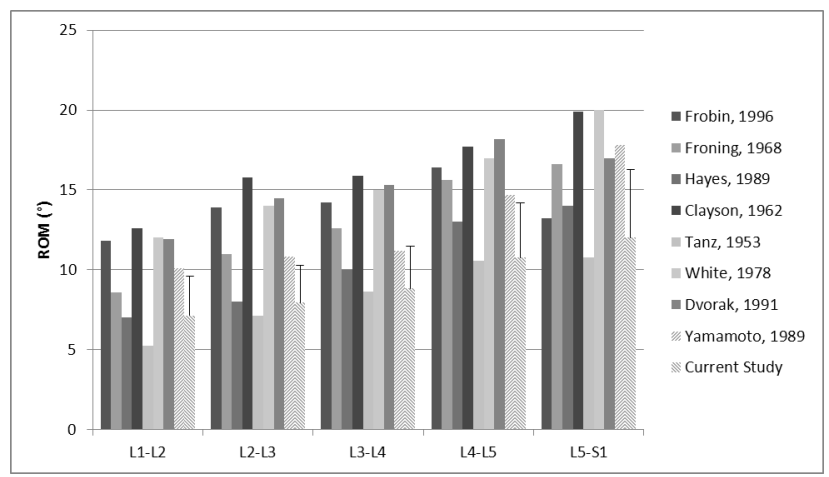

Fig. 7. LB ROM Literature Values Compared to Current Study. Striped bars signify in vitro studies, while shaded ones represent in vivo. Error bars represent standard deviation. persist, indicating that parameters more specific to the morphology and material characteristics of male and female spines must be investigated to explain the observed differences.

Although a detailed characterization of the ROM of the lumbar spine may not contribute significantly to the design and evaluation of devices intended to provide intervertebral fixation, such information may prove to be indispensable in the development of motion preservation devices since the primary design objective of these implants is to restore normal motion. The results of this study indicate that considerable differences exist in the kinematic response of the lumbar FSU depending on the intervertebral level and the gender of the donor. While further study is required to establish the clinical relevance of these differences, it is reasonable to postulate that the design of motion preservation devices should include consideration for the operative level and for the gender of the patient.

Further study is warranted to explain the inter-level and inter-gender differences found in this study. It seems likely that geometrical differences between intervertebral levels and between males and females contribute significantly to the differences observed in this study. For instance, the change in the cross-sectional shape of the lumbar intervertebral disc, which increases in width and decreases in depth down the spinal column, may explain the decrease in sagittal

\begin{tabular}{|c|c|c|c|c|c|c|}
\hline Study & Year & $\begin{array}{l}\text { Symptomatic/ } \\
\text { Asymptomatic }\end{array}$ & $\begin{array}{r}\text { Total } \\
\text { Sample } \\
\text { Size }\end{array}$ & Males & Females & $\begin{array}{r}\text { Age } \\
\text { Range } \\
\text { (yrs) }\end{array}$ \\
\hline Tanz & 1953 & Asymptomatic & 55 & 17 & 38 & $\begin{array}{r}10 \text { Chil- } \\
\text { dren, } 45 \\
\text { Adults } \\
(2-77 \\
\text { years })\end{array}$ \\
\hline Clayson & 1962 & Asymptomatic & 26 & 0 & 26 & $\begin{array}{r}\text { College } \\
\text { Age }\end{array}$ \\
\hline Froning & 1968 & $\begin{array}{r}\text { Symptomatic } \\
\text { but "Normal" } \\
\text { by authors' } \\
\text { definition }\end{array}$ & 30 & unknown & unknown & unknown \\
\hline Hayes & 1986 & Asymptomatic & 59 & 59 & 0 & $19-59$ \\
\hline Dvorak & 1991 & Asymptomatic & 41 & 23 & 18 & $22-50$ \\
\hline Frobin & 1996 & Asymptomatic & 61 & 45 & 16 & $19-57$ \\
\hline
\end{tabular}


plane stiffness from L1-2 to L5-S1. The influence of FSU dimensions, such as disc width, depth and height, on the ROM response should be evaluated in order to establish whether the difference observed between males and females can be explained by the greater size of male segments or whether inherent disparities in material properties govern the observation. In addition, inter-laboratory corroboration of the results presented here will aid in establishing the generalizability of the observed differences by confirming that they are not a result of the testing method.

\section{Conclusion}

To the authors' knowledge this is the first analysis of the in vitro characteristics of the Intact human lumbar spine with such a large sample size. The significant differences in lumbar ROM between male and female spine segments and between the intervertebral levels must be taken into account in study design in order to prevent biases in outcomes. These findings may also have critical implications in the design of spinal implants, particularly those designed to maintain or restore healthy motion. The significant correlations between height, weight and ROM are quite weak $\left(R^{2}=0.049-0.185\right)$ and may be explained by intra-gender differences.

\section{References}

1. White AA, Panjabi MM. The basic kinematics of the human spine. A review of past and current knowledge. Spine (Phila. Pa. 1976). 1978;3:12-20.

2. Nachemson A, Schultz A, Berkson M. Mechanical properties of human lumbar spine motion segments: influences of age, sex, disc level, and degeneration. Spine (Phila. Pa. 1976). 1979;4:1-8.

3. Fujiwara A, Lim TH, An HS, et al. The effect of disc degeneration and facet joint osteoarthritis on the segmental flexibility of the lumbar spine. Spine (Phila $\mathrm{Pa}$ 1976) 2000;25(23):3036-3044.

4. Mimura M, Panjabi MM, Oxland TR, Crisco JJ, Yamamoto I, Vasavada A. Disc degeneration affects the multidirectional flexibility of the lumbar spine. Spine (Phila. Pa. 1976). 1994;19:1371-1380.
5. Yamamoto I, Panjabi MM, Crisco T, Oxland T. Three-dimensional movements of the whole lumbar spine and lumbosacral joint. Spine (Phila. Pa. 1976). 1989;14:1256-1260.

6. Jegapragasan M, Cook DJ, Gladowski DA, Kanter AS, Cheng BC. Characterization of articulation of the lumbar facets in the human cadaveric spine using a facet-based coordinate system. Spine J

11(4):340-346.

7. Yu AK, Siegfried CM, Chew B, et al. Biomechanics of Posterior Dynamic Fusion Systems in the Lumbar Spine: Implications For Stabilization with Improved Arthrodesis. J Spinal Disord Tech.

8. Cook DJ, Yeager MS, Cheng BC. Interpedicular Travel in the Evaluation of Spinal Implants: An Application in Posterior Dynamic Stabilization. Spine (Phila Pa 1976).

9. Goel VK, Panjabi MM, Patwardhan AG, Dooris AP, Serhan H. Test protocols for evaluation of spinal implants. J. Bone Joint Surg. Am. 2006;88 Suppl 2:103-9.

10. Cunningham BW, Setter JC, Shono Y, McAfee PC. Static and Cyclical Biomechanical Analysis of Pedicle Screw Spinal Constructs. Spine (Phila. Pa. 1976). 1993;18(12).

11. Panjabi MM, Abumi K, Duranceau J, Crisco JJ. Biomechanical evaluation of spinal fixation devices: II. Stability provided by eight internal fixation devices. Spine (Phila. Pa. 1976). 1988;13(10):1135-1140. 12. Panjabi M, Yamamoto I, Oxland T, Crisco J. How does posture affect coupling in the lumbar spine? Spine (Phila. Pa. 1976). 1989;14:1002-1011. 13. Wheeler DJ, Freeman AL, Ellingson AM, et al. Inter-laboratory variability in in vitro spinal segment flexibility testing. J. Biomech. 2011;44(13):2383-2387. 14. Panjabi MM, Oxland TR, Yamamoto I, Crisco JJ. Mechanical behavior of the human lumbar and lumbosacral spine as shown by three-dimensional load-displacement curves Lumbosacral Behavior Spine of the Human Curves * and as Shown by Three-Dimensional. 2010.

15. Patwardhan AG, Havey RM, Meade KP, Lee B, Dunlap B. A Follower Load Increases the Load-Carrying Capacity of the Lumbar Spine in Compression. 1999;24(10):1003-1009.

16. Crawford NR, Peles JD, Dickman CA. The spinal lax zone and neutral zone: measurement tech- 
niques and parameter comparisons. J Spinal Disord 1998;11(5):416-429.

17. Frobin W, Biggemann M, Brinckmann $P$, Leivseth G. Precision measurement from flexion-extension lumbar spine of segmental motion radiographs of the. 1996;11(8):457-465.

18. Froning EC, Frohman B. Motion of the Lumbosacral Spine after Laminectomy and Spine Fusion. 1968;50(5):897-918.

19. Hayes MA, Howard TC, Gruel CR, Kopta JA. Roentgenographic evaluation of lumbar spine flexion-extension in asymptomatic individuals. Spine (Phila. Pa. 1976). 1989;14:327-331.

20. Clayson SJ, Newman IM, Debevec DF, Anger RW, Skowlund H V, Kottke F. Evaluation of mobility of hip and lumbar vertebrae of normal young women. Arch. Phys. Med. Rehabil. 1962;43:1-8.

21. Dvorák J, Panjabi MM, Chang DG, Theiler R, Grob D. Functional radiographic diagnosis of the lumbar spine. Flexion-extension and lateral bending.
Spine (Phila. Pa. 1976). 1991;16:562-571.

22. Tanz SS. Motion of the lumbar spine; a roentgenologic study. Am. J. Roentgenol. Radium Ther. Nucl. Med. 1953;69(3):399-412.

\section{Disclosures}

The authors declare no relevant disclosures.

\section{Corresponding Author}

Boyle C. Cheng, Allegheny Health Network, Department of Neurosurgery, 420 East North Ave, Suite 302, Pittsburgh, PA 15212. boylecheng@yahoo.com

Published 5 March 2015.

Copyright @ 2015 ISASS - International Society for the Advancement of Spine Surgery. To see more or order reprints or permissions, see http://ijssurgery.com. 